\title{
Electrical capacitance tomography for flow imaging: system model for development of image reconstruction algorithms and design of primary sensors
}

C.G. Xie

S.M. Huang

B.S. Hoyle

R. Thorn

C. Lenn

D. Snowden

M.S. Beck

\begin{abstract}
A software tool that facilitates the development of image reconstruction algorithms, and the design of optimal capacitance sensors for a capacitance-based 12-electrode tomographic flow imaging system are described. The core of this software tool is the finite element (FE) model of the sensor, which is implemented in OCCAM-2 language and run on the Inmos T800 transputers. Using the system model, the in-depth study of the capacitance sensing fields and the generation of flow model data are made possible, which assists, in a systematic approach, the design of an improved image-reconstruction algorithm. This algorithm is implemented on a network of transputers to achieve a real-time performance. It is found that the selection of the geometric parameters of a 12-electrode sensor has significant effects on the sensitivity distributions of the capacitance fields and on the linearity of the capacitance data. As a consequence, the fidelity of the reconstructed images are affected. Optimal sensor designs can, therefore, be provided, by accommodating these effects.
\end{abstract}

\section{Introduction}

The 8-electrode capacitive tomography method for twocomponent flow imaging has been developed over the past few years and has shown promising applications for multicomponent flow measurement [1-4]. To expand the applicability of this technique, improvements on the

Paper 8390G (E3, E10), received 4th July 1991

C.G. Xie, S.M. Huang and M.S. Beck are with the Department of Electrical Engineering and Electronics, UMIST, PO Box 88, Manchester M60 1QD, United Kingdom

B.S. Hoyle is with the Department of Electrical and Electronic Engineering, University of Leeds, Leeds, LS2 9JT, United Kingdom

R. Thorn is with the School of Electronic Engineering, University of South Australia, Adelaide, South Australia

C. Lenn is with Schlumberger Cambridge Research Ltd., PO Box 153, Cambridge, CB3 OHG, United Kingdom

D. Snowden is with Schlumberger Industries Ltd., Farnborough, Hampshire, GU14 7PW, United Kingdom

IEE PROCEEDINGS-G, Vol. 139, No. 1, FEBRUARY 1992 spatial resolution of the system must be made. To achieve this objective, first, the number of electrodes around the periphery of a pipe should be increased. The number of extra electrodes that can be added is governed by the capability of the transducer hardware in resolving very small capacitances. The improved design of the capacitance transducer electronics has enabled the use of a 12-electrode capacitive sensor [5]; this will more than double the independent capacitance measurements (from 28 to 66 , see eqn. 1), which means that twice the number of objects in the field of view can be resolved.

The second aspect of enhancing the image resolution which forms the focal point of this paper, is to improve the image reconstruction algorithm, which is, as will become clear later, strongly related to the design of a primary capacitance sensor. A poorly designed capacitance sensor can produce extremely nonlinear measurement data, leading to erroneous reconstructed images. The reconstruction algorithm should also be efficient so that a real-time performance (say 20 frames per second) can be realised on a network of transputers.

The aim of the work presented in this paper is to develop a software environment, as shown in Fig. 1, facilitating the design of an image reconstruction algorithm and the optimisation of a 12-electrode primary sensor for a six inch gas/oil pipeline. The core of this software environment is the finite element (FE) model of the sensor system; it is capable of calculating the field sensitivity information needed by the image reconstruction process, and also produces simulated capacitance measurement data for predicting the performance of a complete capacitance tomographic system.

\section{Finite element model : the forward problem}

\subsection{Mathematical formulation of the sensor model} In this study, a two-dimensional (2D) FE model of the sensor system is described. This confines us to deal with an object which is translationally uniform. The calculated capacitance is in units of $F \mathrm{~m}^{-1}$. The model is described for gas/oil two-component flow, however, other twocomponent flows can be treated similarly.

A 12-electrode capacitance sensor is shown in Fig. 2. To establish its mathematical model, it is necessary to 
describe how the capacitance measurements are performed (for detailed description see Reference 5). In a complete measurement cycle, electrode 1 is first supplied with a positive potential $V_{c}$ (which is referred to as the

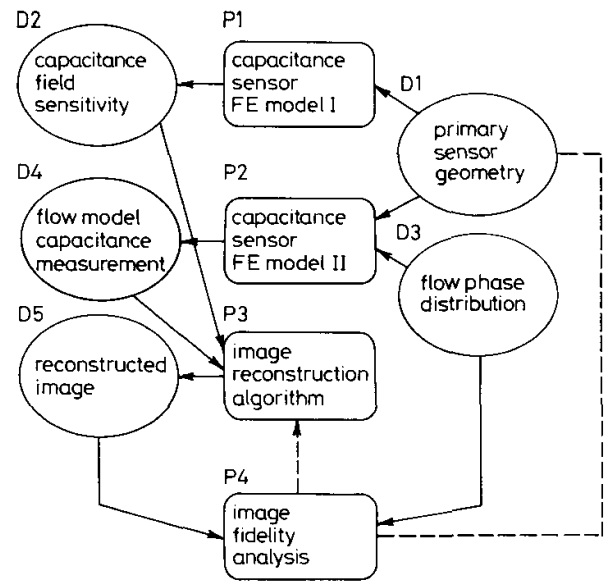

Fig. 1 Flow chart of the software environment facilitating algorithm development and sensor design for electrical capacitive tomography systems

$$
\begin{array}{cc}
\longleftarrow & \text { data flow } \\
\hdashline P_{n} & \text { feedback } \\
\square & \text { process } n \\
D m & \\
\bigcirc & \text { i/o data } m
\end{array}
$$

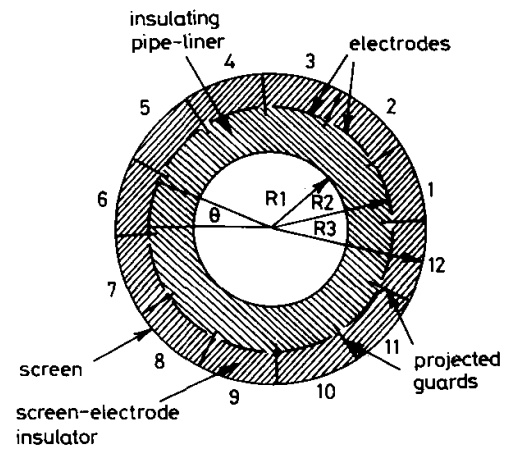

insulator

Fig. 2 Schematic diagram of a 12-electrode capacitive primary sensor

source electrode), and the capacitances between electrodes 1 and 2,1 and $3, \ldots, 1$ and 12 are measured, in parallel, by 11 capacitance transducers; during this measurement phase, electrodes 2 to 12 are at the virtual earth potential imposed by the transducer [5] and they are called the detecting electrodes. Next, electrode 2 is the source electrode and 10 parallel capacitance measurements are performed by the detecting electrodes 3 to 12 electrode 1 , in this measurement phase, because of reciprocity, is the redundant electrode, which is earthed and is called the guard electrode. This process continues until electrode 11 is the source electrode, electrode 12 the detecting electrode, and electrodes 1 to 10 the guard electrodes. The system screen and the projected guards are always maintained at earth potential. Therefore, a total of 66 independent measurements are produced. In general, for an $\mathrm{N}$-electrode system, the number of inde- pendent capacitance measurements $M$ is given by

$$
M=N(N-1) / 2
$$

Since the measurement frequency of the capacitance transducer used is about $1 \mathrm{MHz}$, modelling of the 12 electrode capacitance sensor can be treated as an electrostatic field problem, and can be characterised by Poisson's equation (assuming no free charge),

$$
\nabla \cdot\left[\varepsilon_{0} \varepsilon(x . y) \nabla \phi(x, y)\right]=0
$$

and the associated boundary conditions (the Dirichlet boundary conditions) imposed by this measurement technique, when electrode $i$ is the source electrode $(i=1$, $2, \ldots, 11)$, are

$$
\psi^{(i)}= \begin{cases}V_{c} & (x, y) \subseteq \Gamma_{i} \\ 0 & (x, y) \subseteq \text { all } \Gamma_{k}(k \neq i) \text { and }(x, y) \subseteq\left(\Gamma_{s}+\Gamma_{p g}\right)\end{cases}
$$

where $\phi(x, y)$ and $\varepsilon(x, y)$ are, respectively, the twodimensional potential and dielectric constant distributions, and $\varepsilon_{0}$ the free-space permittivity. $\Gamma_{1}, \Gamma_{2}, \ldots$, and $\Gamma_{12}$ represent the spatial locations of the 12 electrodes, $\Gamma_{s}$ that of the sensor screen, and $\Gamma_{p g}$ that of the 12 projected guards (Fig. 2).

The forward problem is to determine the 66 capacitance measurements for a known distribution of dielectric constant, which, in our case, is the distribution of gas $\left(\varepsilon=\varepsilon_{o a s}=1\right)$ and oil $\left(\varepsilon=\varepsilon_{o i l}=3\right)$ components. To achieve this, eqn. 2 must first be solved to obtain the potential distribution $\phi(x, y)$. Since flow distribution $\varepsilon(x$ $y$ ) is, in general, very irregular, there is no analytical solution to eqn. 2. Therefore, a numerical method based on the finite element method (FEM) is used in this study.

To obtain a FE solution, the region shown in Fig. 2 is divided into $\boldsymbol{P}$ four-noded quadrilateral elements corresponding to $Q$ nodes. The unknown gas/oil distribution can be expressed by a vector $\varepsilon^{*}$, which is the subset of the vector $\varepsilon=\left(\varepsilon_{1}, \varepsilon_{2}, \ldots, \varepsilon_{P}\right)$, representing the dielectric constants of the $P$ elements. When electrode $i$ is the source electrode $(i=1,2, \ldots, 11)$, the resulting nodal potentials are represented by a $Q \times 1$ vector $v^{(i)}$, and it can be shown that $[6,7]$

$$
A v^{(i)}=b^{(i)}
$$

where $b^{(i)}$ is a $Q \times 1$ vector incorporating the boundary conditions expressed by eqn. 3 , and $A$ a sparse $Q \times Q$ matrix whose entries depend on the element dielectric constants $\varepsilon$ and therefore the gas/oil distribution $\varepsilon^{*}$, as well as on the element topology. Since matrix $A$ is symmetric and positive-definite, there exists a nonsingular upper triangular matrix $\boldsymbol{U}$, with unit diagonal entries, and a diagonal matrix $D$, such that $[7]$

$$
\boldsymbol{A}=\boldsymbol{U}^{\boldsymbol{T}} \boldsymbol{D} \boldsymbol{U}
$$

After performing the matrix factorisation indicated by eqn. $5 a$ (this is only done once for all of the 11 different source electrodes as indicated by eqn. 3), the solution to eqn. 4 is carried out in the following three steps [7]:

(a) forward reduction: $U^{T} z=b^{(i)}$

(b) diagonal scaling: $\boldsymbol{D} \boldsymbol{y}=\boldsymbol{z}$

(c) back substitution: $U \boldsymbol{v}^{(i)}=\boldsymbol{y}$

It is worth mentioning that, for a system with a large number of nodes, the dominant portion of computer storage (RAM) is usually that devoted to the matrix $A$. For example, the FE mesh that we used for the 12- 
electrode system has about 2000 nodes; to store matrix $A$ (as single precision 32-bit data), it demands about 16 Mbytes of memory. Since matrix $\boldsymbol{A}$ is very sparse (i.e. it has many zero entries) and is often banded, it can be stored in two vectors (a real and an integer) in a compacted fashion containing fewer entries using the 'skyline' scheme $[6,7]$. In doing so, the storage needed, for a 2000-node system, is usually less than 1 Mbyte, which is well within the memory limit of today's powerful microcomputers.

After solving node potentials from eqns. 4 and 5 , the capacitance of the electrode pair $i-j$, can be calculated by performing the following line-integration numerically:

$$
\begin{aligned}
C_{i, j} & =Q\left(\Gamma_{j}\right) / V_{c} \\
& =\left(\varepsilon_{0} / V_{c}\right) \int_{(x, y) \subseteq \Gamma_{j}} \varepsilon(x, y) \nabla \phi^{(i)}(x, y) \cdot \overrightarrow{d \Gamma}_{j}
\end{aligned}
$$

where $Q\left(\Gamma_{j}\right)$ is the charge sensed by detecting electrode $j$ $(j=i+1, \ldots, 12)$, and $\phi^{(i)}(x, y)$, represented by $v^{(i)}$ in a discrete form, is the potential distribution when electrode $i$ is the source electrode and when a flow $a(x, y)$, represented by vector $\varepsilon$ (and therefore $\varepsilon^{*}$ ) in a discrete form, is present.

The FE model of the 12-electrode system is implemented in OCCAM-2 language running on Inmos T 800 transputers [8]. This makes it easy to integrate the system model with the image reconstruction process for algorithm development and testing (Section 3). The proved image reconstruction algorithm can then be implemented on several transputers to realise real-time performance (Section 6). In this research, an Inmos BOO4-compatible PC add-in board, on which there is a T800 $20 \mathrm{MHz}$ transputer with 4 Mbyte dynamic RAM, is used to perform the FE calculations, as well as serving as the transputer development system (TDS) [9]. For a 2000-node system model, the transputer completes the 66 capacitance calculations $\left(C_{i, j}, i=1,2, \ldots, 11, j=i+1\right.$, $\ldots, 12)$ within 90 seconds, which is considered fast enough for our modelling purposes. Obviously, this calculation time can be reduced by distributing the FE calculation processes over several transputers running in parallel.

\subsection{FE model I: calculation of capacitance field} sensitivity

Two FE models (processes $P 1$ and $P 2$ ) have been established (see Fig. 1). Process $P 1$ calculates the field sensitivity (D2) needed by the image reconstruction process $(P 3)$, for a primary sensor having the geometrical and physical data $D 1$. With reference to Fig. 2 , data $D 1$ include $R_{1}, R_{2}, R_{3}, \theta, d, \varepsilon_{s e}, \varepsilon_{p l}, \varepsilon_{g a s}$ and $\varepsilon_{o i l}$, where $d$ is the length of the projected guard, $\varepsilon_{s e}$ and $\varepsilon_{p l}$ are, respectively, the dielectric constants of the screen-electrode insulator and the pipe-liner.

The field sensitivity distribution of electrode pair $i-j$, $S_{i, j}(k)$, is defined as

$$
S_{i, j}(k)=\mu(k)\left(C_{i, j}(k)-C_{i, j(\text { ass })}\right) /\left(\Delta C_{i, j} \Delta \varepsilon\right)
$$

where

$$
\begin{aligned}
\Delta C_{i, j} & =C_{i, j(o i l)}-C_{i, j(g a s)} \\
\Delta \varepsilon & =\varepsilon_{o i l}-\varepsilon_{g a s}
\end{aligned}
$$

and where $C_{i, f}(k)$ is the capacitance when the $k$ th in-pipe element (the element inside the circle with radius $R_{1}$ ) has dielectric constant $\varepsilon_{o i l}$ and the rest of the in-pipe elements all have that of $\varepsilon_{\text {gas }}$ (in our model, there are 900 in-pipe elements, i.e. the vector $z^{*}$ has 900 entries; $k=1,2, \ldots$, 900). $C_{i, j(a s)}$ and $C_{i, j(o i n)}$ are, respectively, the capacitances when the pipe is filled with gas and with oil; $\Delta C_{i, j}$ is, therefore, the fullscale capacitance change. Note that in this paper $C_{i, j(g a s)}$ are called the system standing capacitances. $\mu(k)$ is a correction factor related to the area of the $k$ th in-pipe element; $\mu(k)$ has different values for elements of different areas.

In process $P 1$, owing to the property of symmetry, only 6 out of 66 distributions of field sensitivity need calculating; they are $S_{1,2}$ (sensitivity distribution of an adjacent electrode-pair, type 1), $S_{1,3}$ (that of an electrodepair separated by one electrode, type 2), $S_{1,4}$ (that of an electrode-pair separated by two electrodes, type 3 ), $S_{1,5}$ (that of an electrode-pair separated by three electrodes, type 4), $S_{1,6}$ (that of an electrode-pair separated by four electrodes, type 5) and $S_{1,7}$ (that of a diagonally separated electrode-pair, type 6 ). This can significantly reduce computation time since the rest of the 60 sensitivity distributions can be obtained by simple rotation transformation (discussed below). To make these six sensitivity distributions accessible by the image reconstruction process $P 3$, a procedure is used in $P 1$ to transform them from the FE domain (which has 900 in-pipe elements of different areas distributed irregularly) to the image domain (which has 812 in-pipe square picture-elements or pixels having the same areal, i.e.

$$
\Omega_{i, j}(p)=T\left\{S_{i, j}(k)\right\} \quad(i=1 \text { and } j=2,3, \ldots, 7)
$$

where $\Omega_{i, j}(p)$ are the transformed sensitivity distributions in the image domain and $p$ the pixel number $(p=1,2$, $\ldots, 812) ; T\{\cdot\}$ denotes the transformation operator. The linear transformations involved are scaling (the sensor geometry has different units in two domains), translation and reflection (if different co-ordinate systems are used in
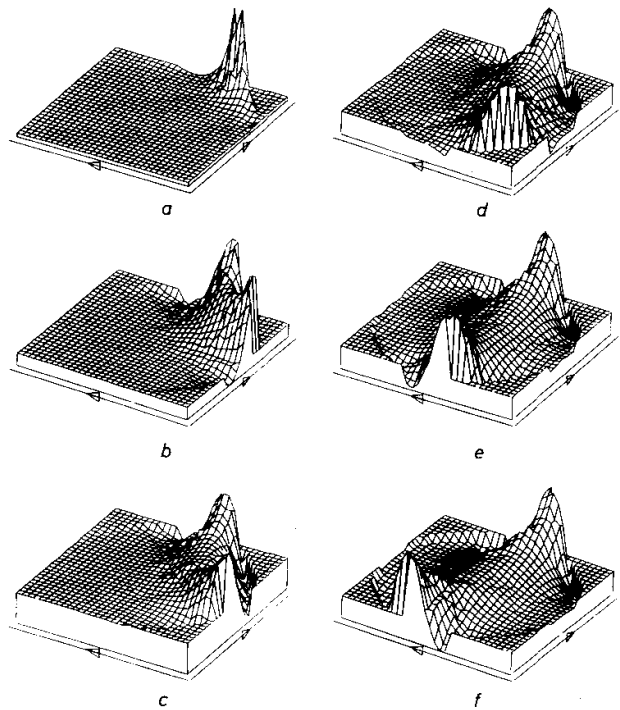

Fig. 3 Capacitance sensitivity distributions of six typical electrode pairs calculated for sensor 2

$R_{1}=76.2 \mathrm{~mm}, R_{2}-R_{1}=15 \mathrm{~mm}, R_{3}-R_{2}=7 \mathrm{~mm}, d=9 \mathrm{~mm}, \theta=26^{\circ}, \varepsilon_{s c}=4$, $\varepsilon_{p i}=5.8, \varepsilon_{g a s}=1$ and $\varepsilon_{o i t}=3$.

$a$ adjacent electrode pair, $\Omega_{1.2}$

$b$ electrode pair separated by one electrode, $\Omega_{1,3}$

$c$ electrode pair separated by two clectrodes, $\Omega_{1,4}$

$d$ electrode pair separated by three electrodes, $\Omega_{1}$,

$f$ diagonally separated electrode pair, $\Omega_{1,7}$ 
two domains), and interpolation or extrapolation of sensitivity values for each pixel $p$.

Fig. 3 shows the typical six transformed sensitivity distributions $\Omega_{1,2}$ to $\Omega_{1,7}$ (type 1 to type 6), for a sensor (sensor 2 in Section 4.2) with $R_{1}=76.2 \mathrm{~mm}, R_{2}-R_{1}=$ $15 \mathrm{~mm}, R_{3}-R_{2}=7 \mathrm{~mm}, d=9 \mathrm{~mm}, \theta=26^{\circ}, \varepsilon_{s e}=4$ and $\varepsilon_{p l}=5.8$. It shows that the field sensitivity is higher near the pipe wall than in the middle of the pipe, and in some areas the sensitivity exhibits positive response, otherwise it is negative (the sunken areas) or zero. The area of positive response interrogates different parts of the pipe cross-section for different electrode pairs.

To obtain the other 60 sensitivity distributions, in process $P 1$, rotation transformations are further employed in the image domain (the centre of the pipe is the centre of rotation), i.e.

$$
\boldsymbol{\Omega}_{m, n}=R\left\{\boldsymbol{\Omega}_{i, j} ; \gamma\right\}
$$

where $R\{\cdot\}$ is an operator representing rotation transformation. Eqn. $7 e$ is interpreted as that $\Omega_{m, n}$ is obtained by rotating $\Omega_{i, j}$ anticlockwise by $\gamma$ degree. Note that $\boldsymbol{\Omega}_{m, n}$ and $\Omega_{i, j}$ must be of the same type. Table 1 gives a full list of the relationship associated with eqn. $7 e$.

\subsection{FE model II: simulation of capacitance} measurements due to various gas/oil flow distributions

Various gas/oil distribution models $D 3$, represented by $\varepsilon(x, y)$ or $\varepsilon$ (and therefore $\varepsilon^{*}$ ) in eqn. 6 , are generated by a different FE model (process P2, see Fig. 1). The corresponding responses of a sensor (with geometrical data $D 1$ ) namely, the 66 capacitance data $(D 4)$, are then calculated by process $P 2$, serving as the flow 'measurement' data for the image reconstruction process $P 3$ (Section 3). In process $P 2$, realistic flow models of different 'regimes', such as stratified flow, core flow, annular flow, gasbubble flow and oil-droplet flow, can be produced by assigning the corresponding entries of the vector $\varepsilon^{*}$ with either $\varepsilon_{\text {gas }}$ or $\varepsilon_{\text {oil }}$. Flows of different volume fractions of oil $(\beta)$, various orientations of gas/oil interfaces in the case of stratified flows, different numbers and sizes of bubbles or droplets can be generated.

Fig. $4 a$ shows an example of a stratified gas/oil flow (data $D 3$ in Fig. 1), where the oil phase is represented by the high grey level $(\beta=0.603)$ and the gas phase by the low one. Fig. $4 b$ shows the corresponding 66 normalised capacitances $\lambda_{i, j}$ (data $D 4$ ), calculated for the primary sensor 2 (Section 4.2). The normalised capacitance $\lambda_{i, j}$ is defined as

$$
\lambda_{i, j}=\left(C_{i, j}^{m}-C_{i, j(g a s)}\right) / \Delta C_{i, j}
$$

where $C_{i, j}^{m}$ is the capacitance measurement due to a gas/oil distribution model. For a linear system, $0 \leqslant$ $\lambda_{i, j} \leqslant 1$. Fig. $4 b$ indicates that, there may be $\lambda_{i, j}<0$ (called 'under-shooting') or $\lambda_{i, j}>1$ (called 'overshooting'). The magnitude of overshooting is, in general, larger than that of the undershooting. This nonlinearity in the capacitance data is caused by the effects of the guard electrodes and the discontinuity in the distribution

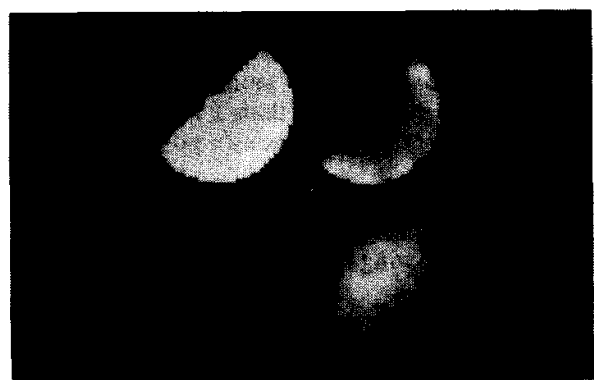

Fig. 4 Typical display on the transputer graphics monitor $a$ a stratified gas/oil flow model, where the high grey level represents the oil phase and the low the gas

$b$ the corresponding 66 normalised capacitances $\dot{\lambda}_{i, j}$ of flow model (a) calculated for sensor 2

$c$ image reconstructed using $\lambda_{i, j}$ and the improved backprojection algorithm using full sensitivity data as shown in Fig. 3

$d$ image reconstructed using the ' $0 / 1$ algorithm'

of dielectric constants $\varepsilon^{*}$. With respect to the situations when there is only gas or oil in the pipe, the presence of a gas/oil mixture will redistribute the electric flux lines in the system. This redistribution may cause some detecting electrodes to absorb more (or less) electric flux lines than when the pipe is filled with oil (or gas), resulting in overshooting (or undershooting) effects.

\section{Image reconstruction: the inverse problem}

For a capacitance tomography system, the inverse problem is to determine the distribution of dielectric constants of gas/oil components (the vector $\varepsilon^{*}$ ) from the measured 66 capacitances $C_{i, j}$, i.e. to find the inverse of eqn. 6 . It should be pointed out that there is no exact/ analytical solution to such an inverse problem.

Important features associated with the capacitance sensing field are that, the electric flux lines, expressed by field vector $\varepsilon(x, y) \nabla \phi(x, y)$, tend to spread, and worst of all, are related to the object $\varepsilon(x, y)$ to be imaged (also see eqns. 2 and 6). This is distinctly different from other tomography techniques, such as the $\mathrm{X}$-ray CT, where the source lines pass directly through the object to be imaged. Therefore, we call the capacitance field a 'soft field', which is inherently nonlinear, and the related problems the soft field problems. Because of these features, the image resolution of a capacitance tomography system is poor in comparison with the X-ray CT (the electric flux lines are difficult to focus), and the well developed image reconstruction algorithms for X-ray CT cannot directly be used for capacitance tomography. However, since a capacitance tomography system has very fast response, is

Table 1 : List of other 60 sensitivity distributions $\Omega_{m, .}$ versus the typical 6 ones

\begin{tabular}{|c|c|c|c|c|c|c|c|c|c|c|c|}
\hline \multirow{2}{*}{$\mathbf{\Omega}_{i, j}$} & \multicolumn{11}{|c|}{$\Omega_{m, n}$} \\
\hline & $\Omega_{2,3}$ & $\Omega_{3,4}$ & $\Omega_{4.5}$ & $\mathbf{\Omega}_{5.6}$ & $\Omega_{6,7}$ & $\Omega_{7,8}$ & $\Omega_{\mathbf{8}, 9}$ & $\Omega_{9,10}$ & $\Omega_{10.11}$ & $\Omega_{11,12}$ & $\Omega_{1,12}$ \\
\hline$\Omega_{1,3}$ & $\Omega_{2,4}$ & $\Omega_{3,5}$ & $\Omega_{4,6}$ & $\Omega_{5,7}$ & $\Omega_{6,8}$ & $\Omega_{7,9}$ & $\Omega_{\text {B.10 }}$ & $\mathbf{\Omega}_{\mathbf{9}, 11}$ & $\Omega_{10.12}$ & $\Omega_{1,11}$ & $\Omega_{2,12}$ \\
\hline$\Omega_{1,4}$ & $\Omega_{2,5}$ & $\Omega_{3.6}$ & $\Omega_{4.7}$ & $\Omega_{5.8}$ & $\mathbf{\Omega}_{6.9}$ & $\Omega_{7,10}$ & $\Omega_{8, \cdots}$ & $\Omega_{9,12}$ & $\Omega_{1,10}$ & $\Omega_{2,11}$ & $\Omega_{3,12}$ \\
\hline$\Omega_{1,5}$ & $\Omega_{2,6}$ & $\mathbf{\Omega}_{3,7}$ & $\Omega_{4,8}$ & $\Omega_{5,9}$ & $\mathbf{\Omega}_{6,10}$ & $\Omega, 1$ & $\Omega_{\text {8.12 }}$ & $\Omega_{1,9}$ & $\Omega_{2,10}$ & $\Omega_{3,11}$ & $\Omega_{4.12}$ \\
\hline$\Omega_{1,6}$ & $\Omega_{2.7}$ & $\Omega_{3, \text { B }}$ & $\Omega_{4.9}$ & $\Omega_{5,10}$ & $\Omega_{6,11}$ & $\Omega_{7,12}$ & $\Omega_{1,8}$ & $\Omega_{2,9}$ & $\Omega_{3.10}$ & $\Omega_{4,11}$ & $\Omega_{5.12}$ \\
\hline$\Omega_{1,7}$ & $\Omega_{2,8}$ & $\mathbf{\Omega}_{3,9}$ & $\Omega_{4,10}$ & $\Omega_{5,11}$ & $\Omega_{6,12}$ & & & & & & \\
\hline . & $30^{\circ}$ & $60^{\circ}$ & $90^{\circ}$ & $120^{\circ}$ & $150^{\circ}$ & $180^{\circ}$ & $210^{\circ}$ & $240^{\circ}$ & $270^{\circ}$ & $300^{\circ}$ & $330^{\circ}$ \\
\hline
\end{tabular}
$\mathbf{\Omega}_{i, j}$ and the corresponding rotation angle $\gamma$ 
inexpensive, compact and nonhazardous, it will no doubt find more widespread use in many industries.

To solve the inverse problem for capacitance tomography, some approximation methods are needed. Note that, in general, the number of unknowns in the system

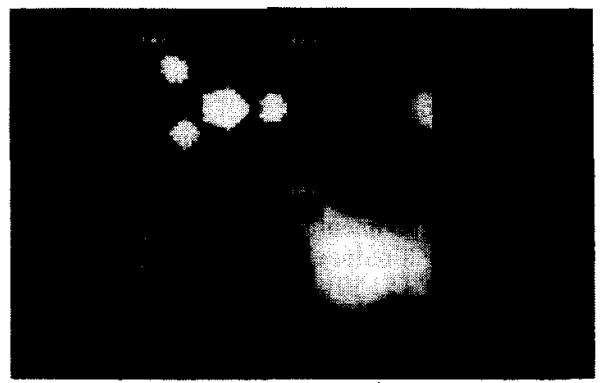

Fig. 5 Typical display on the transputer graphics monitor a a gas/oil distribution model with multiple oil 'droplets' $b \lambda_{i, j}$ of the flow model calculated for sensor 2 c reconstructed image using improved algorithm $d$ that using ' $0 / 1$ algorithm'

(e.g. the 812 image pixels) is more than that of the knowns (e.g. the 66 capacitance measurements), so that the system is underdetermined. Some prior knowledge of the system, such as the field sensitivity distributions, should be used to alleviate the problem. In principle, to correct the field distortions, an iterative method must be used to obtain an accurate solution, however, its implementation often demands intensive computations and, in some cases, convergence cannot be guaranteed. For example, the iterative use of FE calculation can, in principle, find a true dielectric constant distribution, based on appropriate capacitance data. However, for a 2000-node FE problem, it may take one second to complete one iteration even when a network of $100 \mathrm{~T} 800$ transputers is employed. This is, at present costs, a very expensive system for industrial use. Furthermore, the use of such a large transputer network would not have achieved the required real-time performance (say 20 frame images per second). Therefore, a noniterative method, although less accurate, is used.

An image reconstruction algorithm based on a simple backprojection method has been developed in the past for capacitance tomography $[1,2]$. When the backprojection process was carried out, only the binary information of the capacitance sensitivity distributions (as shown in Fig. 3) was used, i.e. $\Omega_{i, j}=1$ if $\Omega_{i, j}>0$, otherwise $\Omega_{i, j}=0$ (this is called the ' $0 / 1$ algorithm' below for brevity). A direct consequence of this method is that the reconstructed image is seriously distorted (see, for example, Fig. $4 d$ ).

To overcome these problems, the backprojection algorithm has to be improved. The modified algorithm uses the full sensitivity information $\boldsymbol{\Omega}_{i, j}$, precalculated as shown in Section 2.2. Let a reconstructed image be represented by pixel grey-level $G(p)$, which is used to approximate the vector $\varepsilon^{*}$ and which can be calculated from the following equations :

$$
G(p)=W(p) \sum_{i=1}^{11} \sum_{j=i+1}^{12} \lambda_{i, j} \Omega_{i, j}(p) \quad(p=1,2, \ldots, 812)
$$

where

$$
W(p)=\left(\sum_{i=1}^{11} \sum_{j=i+1}^{12} \Omega_{i, j}(p)\right)^{-1}(p=1,2, \ldots, 812)
$$

Note that $W(p)$ are the weighting factors used to compensate for the effects of nonuniform sensitivity distribution on the values of pixel grey-level. The plot of $W(p)$ for the primary sensor 2 (Section 4.2 ) is shown in Fig. $6 b$.
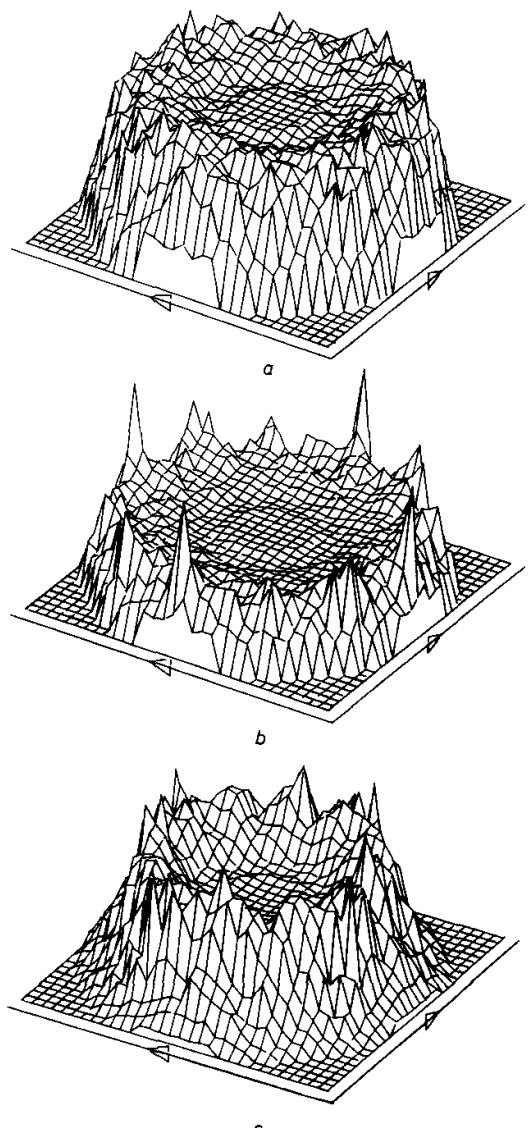

Fig. 6 The distribution of the weighting factors $W(p)$ for pixel greylevels $G(p)(e q n .9 b)$

a sensor 1 with $R_{2}-R_{1}=10 \mathrm{~mm}$

b sensor 2 with $R_{2}-R_{1}=15 \mathrm{~mm}$

$c$ sensor 3 with $R_{2}-R_{1}=20 \mathrm{~mm}$. $d=9 \mathrm{~mm}, \theta=26^{\circ}, \varepsilon_{s e}=4, \varepsilon_{p i}=5.8, \varepsilon_{\text {par }}=1$ and $\varepsilon_{\text {oit }}=3$

For a linear system, the normalised capacitance data $\lambda_{i, j}$ should fall within the interval $[0,1]$, so should the resulting image grey-level $G(p)$. Since a capacitance tomography system exhibits nonlinearity, $\lambda_{i, j}$ may have overshooting (larger than 1) or undershooting (smaller than 0) values (Section 2.3 ), so may $G(p)$. Therefore, some processing on $G(p)$ is needed before the grey-level image is displayed. Overshooting in $G(p)$ is eliminated by using a truncation operation, i.e. in eqn. $9 a$

$$
\lambda_{i, j}=1 \text { if } \lambda_{i, j}>1
$$

Undershooting is dealt with by using a threshold operation described below.

The use of a threshold operation can reduce the low grey-level artefacts present in an image. It has been observed that the level of thresholding is dependent upon the flow distribution and the flow volume fraction, so an 
adaptive threshold operation is required. Numerical experiments have suggested that the following threshold operation is suitable (in our imaging system, 256 integer grey-levels, $0-255$, are used).

$$
G_{t}(p)= \begin{cases}0 & \text { if } G(p)<\eta \\ 255 G(p) & \text { otherwise }\end{cases}
$$

where the threshold level $\eta$ is $(0 \leqslant \eta \leqslant 1)$,

$$
\eta=(1-0.5 \alpha) \zeta
$$

and where

$$
\begin{aligned}
& \alpha=A V G\left(\left|\lambda_{i, j}\right| \quad\left\{\lambda_{i, j}=1 \quad \text { if } \lambda_{i, j}>1\right\}\right) \\
& \zeta=A V G(G(p) \quad \text { for } G(p)>0\})
\end{aligned}
$$

where $A V G(\cdot)$ denotes an average operator and $\{\cdots\}$ a conditional statement.

To illustrate the effectiveness of this algorithm, Fig. $4 c$ shows the reconstructed image (data D5, see Fig. 1) using the capacitance data $D 4$ shown in Fig. $4 b$, which closely resembles the flow model $D 3$ (Fig. $4 a$ ). The image reconstructed using the ' $0 / 1$ algorithm' and a similar threshold operation is shown in Fig. $4 d$; it has poorer fidelity in comparison with Fig. $4 c$ (process $\mathrm{P4}$ in Fig. 1 is involved).

To illustrate the capability of the capacitance tomography system in distinguishing multiple objects, gas/oil flow model depicted in Fig. $5 a$ is used, where the total oil concentration $\beta=0.203$. The corresponding capacitance data $\lambda_{i j}$ from sensor 2 (Section 4.2) are shown in Fig. 5b. From the reconstructed image (Fig. 5c), the presence of four individual 'oil droplets' can hardly be identified, especially the two nearer the centre of the pipe. This indicates the inherent limitation with a capacitance tomography system; it has a position-dependent spatial resolution over the pipe cross-section. When a small object moves from near the pipe wall towards the pipe centre, the images reconstructed change from being sharp to being seriously blurred.

For comparison, the image reconstructed using the '0/1 algorithm' is also displayed (Fig. $5 d$ ), indicating an even poorer distinguishability.

\section{Image fidelity against sensor structure: the} sensor design

\subsection{Sensor design parameters}

The structure of a primary sensor will no doubt influence the performance of a capacitance measurement system. FEM has been used to study the effects of variations of sensor parameters on the capacitance sensitivity distributions and on the system response [10]. The design parameters associated with a 12-electrode sensor are (see Fig. 2)

(a) $R_{1}$ : the pipe radius.

This parameter is fixed for a particular pipe line. For a six inch (I.D.) pipe, $R_{1}=76.2 \mathrm{~mm}$. Other parameters $R_{2}$ $-R_{1}$ and $R_{3}-R_{2}$ discussed below are based on a six inch pipe.

(b) $R_{2}-R_{1}, \varepsilon_{p l}$ : the thickness of pipe-liner with dielectric constant $\varepsilon_{p l}$.

Ideally, $\varepsilon_{p t}$ should be small to minimise the field divergence. However, other factors, such as the requirements of corrosion, abrasion and temperature resistance, and the temperature stability, may limit the selection of the pipe-linear material. The machinable glass (ceramic) used for our sensor has $\varepsilon_{n l}=5.8$.

Small $R_{2}-R_{1}$ would be desirable to save material and, therefore, to reduce the cost of the sensor. However, it should be borne in mind that the requirements of mechanical strength of the sensor to withstand certain pressures and the ease of machining mean that there is a minimum thickness for the pipe-liner (not smaller than $10 \mathrm{~mm}$ in our case). More importantly, $R_{2}-R_{1}$ has a significant effect on the performance of the imaging system and should be carefully selected. This will be illustrated in Section 4.2

(c) $R_{3}-R_{2}, \varepsilon_{s e}$ : the thickness of screen-electrode insulator with dielectric constant $\varepsilon_{s e}$.

An earthed screen is necessary to eliminate the external electrical interferences and to protect electrodes from damage. Small $R_{3}-R_{2}$ would make the sensor compact, but theoretical and experimental studies have found that decreasing $R_{3}-R_{2}$ will reduce the standing capacitances of the sensor system $[5,10]$. It is possible that these capacitances may become too small to measure. Therefore, when selecting this parameter, the measurement resolution of the capacitance transducer hardware must be taken into account. It has been found that $R_{3}-R_{2}=$ $7 \mathrm{~mm}$ is suitable for our sensor and transducer systems.

The selection of $\varepsilon_{s e}$ is not crucial. Potting material is used as the screen-electrode insulator for our sensor, and it has $\varepsilon_{s e}=4$.

(d) $\theta$ : the size of electrode.

For a 12 -electrode system, $\theta$ must be smaller than $30^{\circ}$. A larger $\theta$ will give rise to larger standing capacitances of the sensor (especially those of diagonally separated electrode pairs, their increases would be most beneficial for the capacitance measurement circuit) and higher capacitance sensitivities, and is therefore desirable. To allow some space for the projected guards (see below), $\theta=26^{\circ}$ has been selected for our sensor. The axial length of each electrode is $10 \mathrm{~cm}$.

(e) $d$ : the length of projected guards.

The introduction of projected guards has been found to be an effective means of reducing the standing capacitances of the adjacent electrode-pairs significantly. Note that this will also reduce the standing capacitances of other electrode pairs but to a much lower percentage (Fig. 7). The transducer hardware will benefit most from

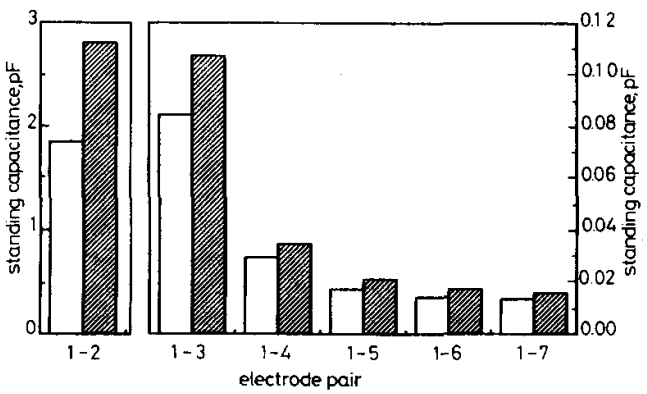

Fig. 7 Calculated standing capacitances of six typical electrode pairs for sensors with projected guards $(d=9 \mathrm{~mm}$, sensor 2$)$ and without pro jected guards $(d=0$, sensor $2 A)$

Note that different vertical scale is used for electrode pair 1-2 Other sensors' parameters are $R_{1}=76.2 \mathrm{~mm}, R_{2}-R_{1}=15 \mathrm{~mm}, R_{3}-R_{2}=$ $7 \mathrm{~mm}, \theta=26^{\circ}, \varepsilon_{\mathrm{sec}}=4, \varepsilon_{\mathrm{pl}}=5.8, \varepsilon_{\mathrm{ew}}=1$ and $\varepsilon_{\mathrm{ail}}=3$ The axial length of each electrode is $10 \mathrm{~cm}$

Sensor 2

Sensor $2 \mathrm{~A}$

this design since a very large standing capacitance would make balancing the capacitance-measuring circuit difficult (in the present circuit design, the maximum standing capacitance allowed is $2 \mathrm{pF}$ [5]). A value of $d=9 \mathrm{~mm}$

IEE PROCEEDINGS-G, VOL. I39, No, I, FEBRUARY 1992 
has been found suitable. It has been observed that the use of projected guards has a minor effect on the fidelity of the reconstructed images.

The electrodes and the projected guards are assumed to be very thin in this FE model. This is justified since in practical use, electrodes and guards are only about $0.1 \mathrm{~mm}-1 \mathrm{~mm}$ thick; their fabrication materials can be brass, stainless steel etc.

The details design of some of the parameters described above has been carried out by Khan and Abdullah using the FEM [11]. We demonstrate below how the variation of pipe-liner thickness $\left(R_{2}-R_{1}\right)$ will affect the linearity of capacitance measurements and the fidelity of reconstructed images.

\subsection{Effects of sensor parameter $R_{2}-R_{1}$ on image} reconstruction

To illustrate the effect of sensor design on image reconstruction, three sensors with different thicknesses of pipeliner are investigated; they are sensor 1 with $R_{2}-R_{1}=$ $10 \mathrm{~mm}$, sensor 2 with $R_{2}-R_{1}=15 \mathrm{~mm}$ and sensor 3 with $R_{2}-R_{1}=20 \mathrm{~mm}$; other sensor parameters are fixed and they are $R_{1}=76.2 \mathrm{~mm}, R_{3}-R_{2}=7 \mathrm{~mm}$, $d=9 \mathrm{~mm}, \theta=26^{\circ}, \varepsilon_{s e}=4$ and $\varepsilon_{p l}=5.8$ (Section 4.1).

Sensitivity distributions $\Omega_{i, j}$ of the three sensors were first calculated (Section 2.2). Fig. 6 shows the plots of weighting factors $W(p)$, calculated from $\Omega_{i, j}$ (eqn. $9 b$ ). Plot $W(p)$ can give an indication of the uniformity of the capacitance sensitivity distribution. More uniform $W(p)$ means that there is a smaller difference between the sensitivities near the pipe wall and in the middle of the pipe, and therefore less correction is needed for the image reconstructed (eqn. $9 a$ ). In this sense, sensor 2 with $R_{2}$ $-R_{1}=15 \mathrm{~mm}$ is a preferred choice. Field uniformity can also be seen from the sensor's fullscale capacitance change, $\Delta C_{i, j}$, shown in Fig. 8 . It can be seen that,

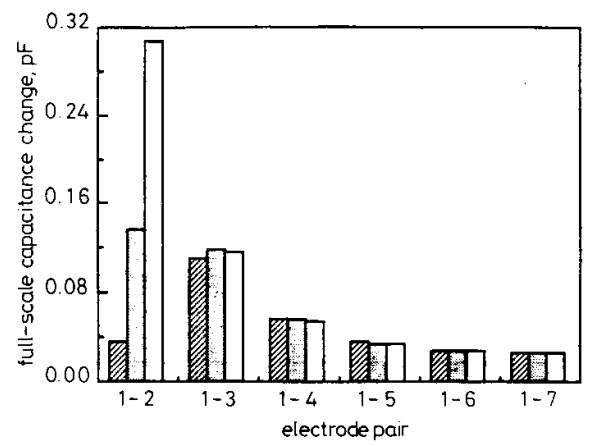

Fig. 8 Calculated full-scale capacitance change of six typical electrode pairs for sensor $1\left(R_{2}-R_{1}=10 \mathrm{~mm}\right)$, sensor $2\left(R_{2}-R_{1}=15 \mathrm{~mm}\right)$ and sensor $3\left(R_{2}-R_{1}=20 \mathrm{~mm}\right)$

Other sensors' parameters are: $R_{1}=76.2 \mathrm{~mm}, R_{3}-R_{2}=7 \mathrm{~mm}, d=9 \mathrm{~mm}$ $\theta=26^{\circ}, \varepsilon_{s e}=4, \varepsilon_{p t}=5.8, \varepsilon_{\text {out }}=1$ and $\varepsilon_{\text {oil }}=$

The axial length of each electrode is $10 \mathrm{~cm}$

Sensor 3

Sensor 3

Sensor 1

$\Delta C_{1,2} / \Delta C_{1,7}$ is 5.2 for sensor 2 , whereas for sensor 1 this ratio is 11.9 , which is more than doubled. For sensor 3 , $\Delta C_{1,2} / \Delta C_{1,7}$ is 1.4 , which is abnormally small and is found to be the cause of severe nonlinearity present in the capacitance data (see below).

Simple flow models can be used to visualise the fidelities of the images reconstructed using these sensors. For

IEE PROCEEDINGS-G, Vol. 139, No. 1, FEBRU ARY 1992 example, Figs. 9.1 $a, 9.2 a$ and $9.3 a$ show the same stratified gas/oil flow model $(\beta=0.20)$ used for this purpose. Fig. 9.1b, 9.2b and $9.3 b$ give the corresponding 66 normalised capacitances $\lambda_{i, j}$ obtained by sensors 1,2 and 3 ,
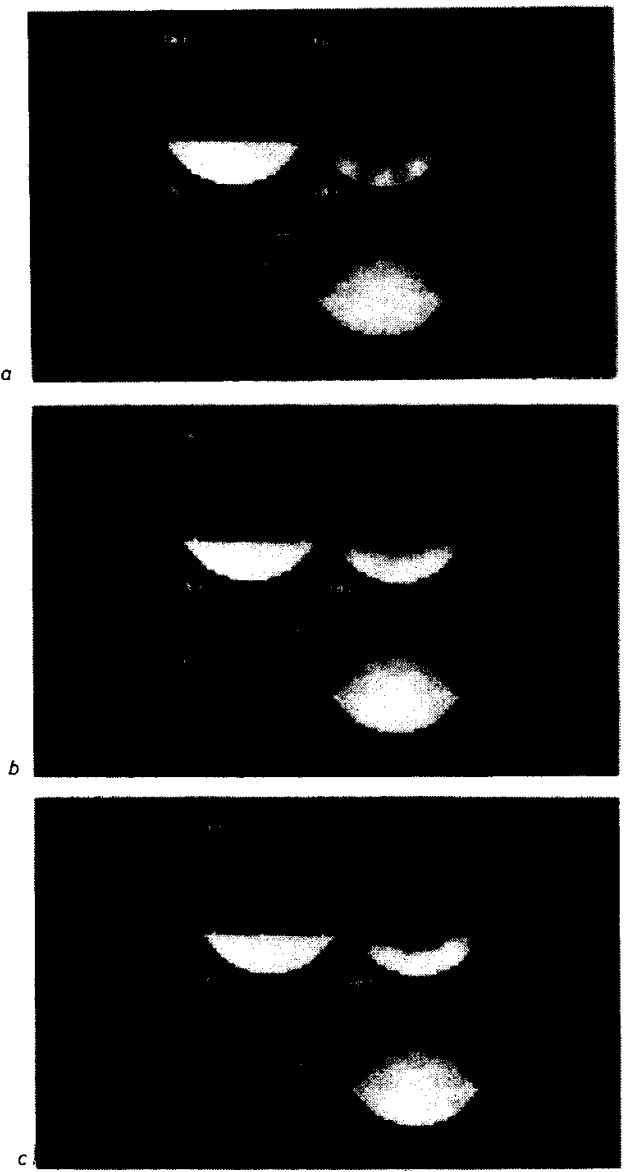

Fig. 9 Image reconstruction of a stratified flow (that shown in all Fig. (a)s) using three different sensors

$a$ sensor 1 with $R_{2}-R_{1}=10 \mathrm{~mm}$

$b$ sensor 2 with $R_{2}-R_{1}=15 \mathrm{~mm}$

$c$ sensor 3 with $R_{2}-R_{1}=20 \mathrm{~mm}$

The rest of the sensors' parameters are $R_{1}=76.2 \mathrm{~mm}, R_{3}-R_{2}=7 \mathrm{~mm}$ $d=9 \mathrm{~mm}, \theta=26^{\circ}, \varepsilon_{s e}=4, \varepsilon_{p t}=5.8, \varepsilon_{\text {gas }}=1$ and $\varepsilon_{o i l}=3$

All figure $(b)$ depict the sensors' capacitance data $\hat{\lambda}_{j, j}$

All figure (c)s show the images reconstructed using the improved backprojection algorithm

All figure ( $d$ )s show those using the ' $0 / 1$ algorithm'

respectively. It can be seen that the capacitance data from sensor 3 exhibit severe nonlinearity (large-scale overshooting and undershooting, see also Fig. 10.3b). This is caused by the selection of the pipe-liner $(20 \mathrm{~mm})$; it is so thick that the sensing fields of adjacent electrode pairs are concentrated mostly in the pipe-liner. This is why $\Delta C_{1,2} / \Delta C_{1,7}$ is abnormally small for sensor 3 (see Fig. 8).

Images reconstructed from the appropriate capacitance data, using the improved backprojection algorithm (Section 3), are shown in Fig. 9.1c, 9.2c and 9.3c. By comparison, the image reconstructed from sensor 3 has the poorest fidelity because of the severe nonlinearity of the capacitance data mentioned above, and that from 
sensor 2 has better fidelity than that from sensor 1 . For these three sensors, images reconstructed using the ' $0 / 1$ algorithm' are also shown in Figs. 9.1d, 9.2d and 9.3d, respectively. These images all have poorer fidelity than
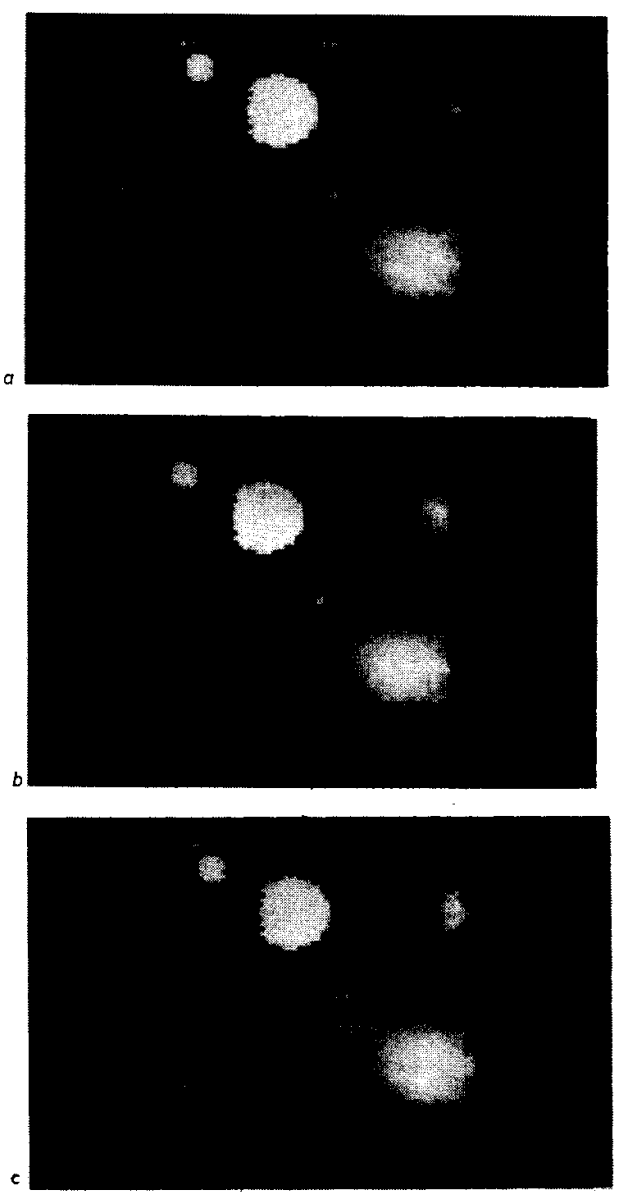

Fig. 10 Image reconstruction of two oil 'droplets' in gas (that shown in all figure (a)s) using three different sensors

a sensor 1 with $R_{2}-R_{1}=10 \mathrm{~mm}$

$b$ sensor 2 with $R_{2}-R_{1}=15 \mathrm{~mm}$

$K_{1}=76.2 \mathrm{~mm}, R_{3}-R_{2}=7 \mathrm{~mm}$

$=9 \mathrm{~mm}, \theta=26^{\circ}{ }^{\circ}=4,0=5.8, \quad 1$ and $R_{1}=3,2 \mathrm{~mm}, R_{3}-R_{2}=7 \mathrm{~mm}$

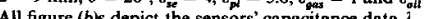

All fgure (c) show the imars reconstructed using ${ }^{j}$ the inproved backprojection

algorith

their counterparts obtained from using the improved algorithm, and they exhibit no significant difference from each other since the sensitivity data of each sensor, after the $0 / 1$ binary operation, have virtually no difference.

Many other flow models have been used to test the performance of these sensors, and it has been found that sensor 2 , in virtually all the cases, delivers the best image fidelity. To reinforce this finding, another gas/oil flow model $(\beta=0.291)$ and the corresponding reconstructed images are given in Fig. 10 .

In summary, from the point of view of high fidelity of reconstructed images, which is the ultimate criterion of any tomography system, sensor 2 with $R_{2}-R_{1}=15 \mathrm{~mm}$ is an optimal design. Note that, for other two-component flows, such as an oil/water flow, this optimal sensor is not necessarily the optimal one. A similar approach can be used to redesign the sensor.
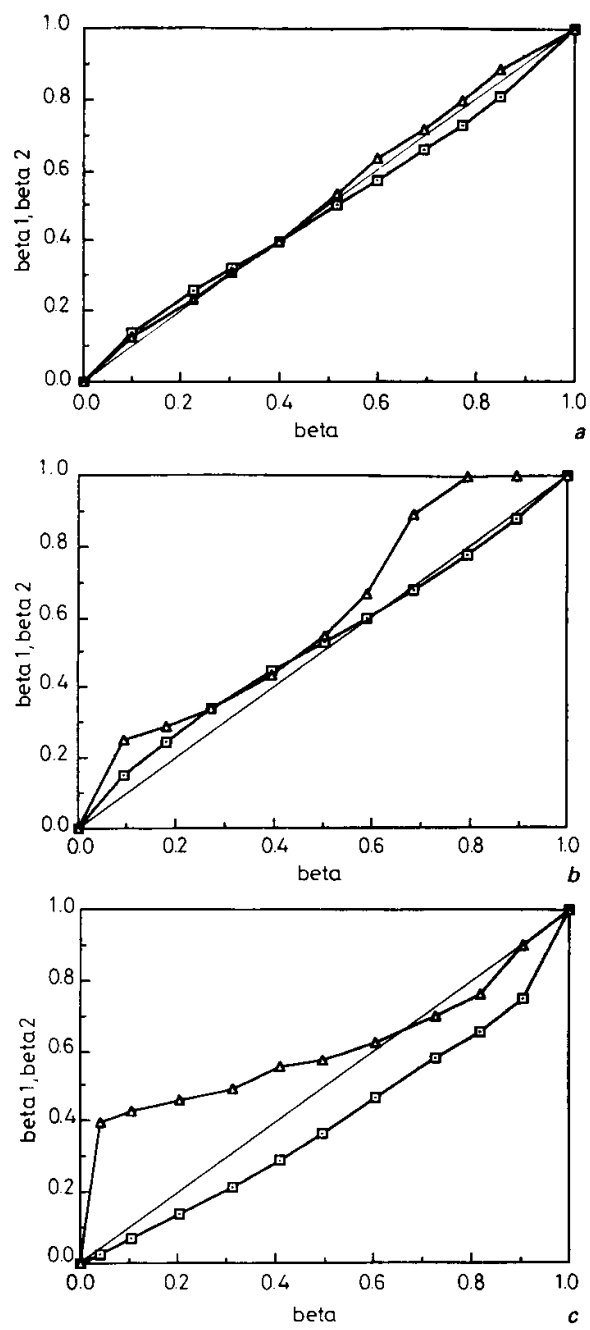

Fig. 11 Oil concentrations calculated from the image $\left(\beta_{1}\right)$ and from the average of the 66 normalised capacitances $\left(\beta_{2}\right)$ against the concentration of the flow models $(\beta)$

a stratified flow

$b$ annular flow

The straight line (with no symbols) shows the ideal relationship

- $-1-$ beta 2

\section{Concentration measurement of flow components}

The simplest way to determine the oil concentration of a gas/oil flow is to sum up all the pixels that have grey levels above the threshold (eqn. 10a); this sum is then divided by the total number of pixels in the pipe ( 812 for our imaging system). The resulting oil concentration is denoted as $\beta_{1}$. The accuracy of this method depends largely on the precise use of the threshold level, which has been found to be difficult to achieve. The results of $\beta_{1}$ against oil concentration of gas/oil flow model $\beta$ are

IEE PROCEEDINGS-G, Vol. 139, No. 1, FEBRUARY 1992 
shown in Fig. 11 for stratified flows, annular flows and core flows. Fig. $11 a$ indicates that the method of determining oil concentration from the image works quite well for stratified flows. However, the results for core flows are the most erroneous (Fig. 11c); because of the inherently low spatial resolution in the centre of the pipe, the images for core flows are blurred and occupy a larger pixel area than the real flows do; consequently, oil concentrations are overestimated, especially at low oil concentrations $(\beta)$. For annular flows (Fig. 11b), the discrepancy between $\beta_{1}$ and $\beta$ is also unacceptably high, in particular at high oilconcentrations.

An alternative, and simpler, method has been used to reduce the measurement error; it uses the average of the 66 normalised capacitance measurements as an indication of oil concentration, denoted as $\beta_{2}$,

$$
\beta_{2}=A V G\left(\left|\lambda_{i, j}\right|\right)
$$

The results of $\beta_{2}$ against $\beta$ are also shown in Fig. 11 for the above three flows. Again, for stratified flows, $\beta_{2}$ and $\beta$ have maintained the closest agreement (Fig. 11a); for annular flows, the use of $\beta_{2}$, instead of $\beta_{1}$, has substantially reduced the measurement error (Fig. 11b). The accuracy for core flows is also improved to a large extent (Fig. 11c), though the oil concentration is underestimated but the monotonic relationship between $\beta_{2}$ and $\beta$ is obtained, making the correction much less difficult.

To increase the accuracy of concentration measurement further, calibrations of the sensor system for various be developed to recognise the various flow regimes from the distribution of the capacitance data, and the corresponding calibration data for the identified flows can then be retrieved.

\section{Parallel implementation of image reconstruction algorithm}

The image reconstruction algorithm described in Section 3 is implemented using a transputer-based system. It takes about $100 \mathrm{~ms}$ for one T800 (25 MHz) transputer to complete one image reconstruction; this is equivalent to flow regimes are needed. An expert system approach is to

an imaging rate of about 15 frames per second. It has been found that nearly three-quarters of this time is spent waiting for values to be fetched from and written to memory, accounting for a great percentage of an application's total running time. The investigation of ways of decreasing memory bottleneck time becomes a developing issue [12].

For our flow imaging system, to increase the imaging speed, multiple transputer-based processors can be used in parallel to implement the backprojection algorithm. One of the simplest, and often the most efficient, ways of exploiting parallel processing is to distribute independent tasks to each of the processors. Such a configuration of the system is often called a task farm [13]. The transputer-based capacitance tomography system is organised in this way as a load-balancing pipeline (Fig. 12). The task farm consists of a master processor (T800 $25 \mathrm{MHz}$ with 1 Mbyte VRAM), whose function is to distribute the tasks (a stream of the 66 capcitance measurements) and collect the results (the corresponding reconstructed images and flow concentrations), and some number of slave processors (T801 $25 \mathrm{MHz}$ typically with 2 Mbyte DRAM), that actually do the work (each slave processor executes the same serial program - the backprojection algorithm - on its own data set). In a loadbalancing pipeline, several buffer processes (the 'throughput' and the 'feedback') carrying tasks to workers, and results to the display are also run on each slave processor, ensuring that whenever a slave processor completes one task another is immediately available. Other processors in the task farm are: (i) a processor (host and monitor) handling the external $\mathrm{I} / \mathrm{O}$; this is a PC-hosted transputer (T800 $20 \mathrm{MHz}$ with 4 Mbyte DRAM on a PC add-in card; Inmos BOO4 compatible and used as TDS), performing the booting of other processors in the network, monitoring (via VDU), keyboard input and loading/saving data from/to the PC hard-disk; (ii) a processor (T222 or T414 $20 \mathrm{MHz}$ with, say, $8 \mathrm{~K}$ SRAM) controlling the remote sensor electronic circuitry [5] which acquires capacitance data needed by the master processor for distributing over the task farm. The task farm

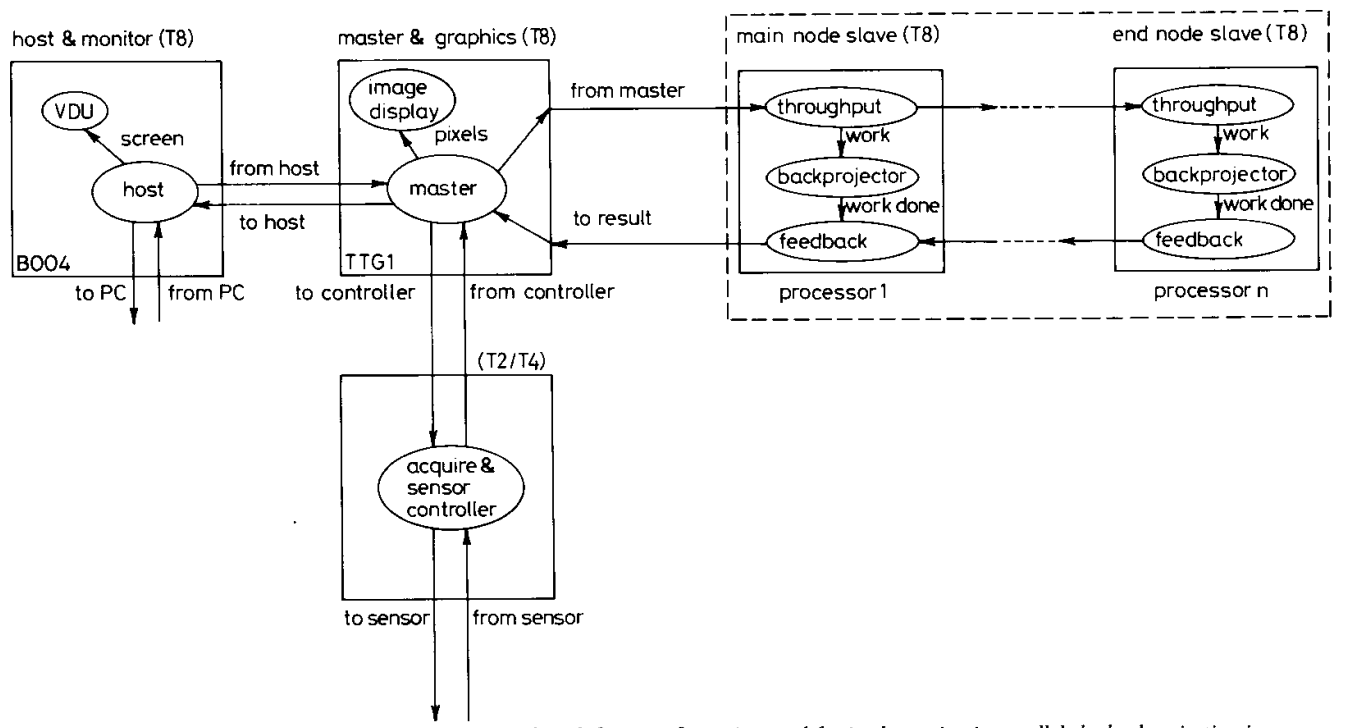

Fig. 12 Transputer-based multi-processor system with task farm configuration used for implementing in parallel the backprojection image reconstruction algorithm

IEE PROCEEDINGS-G, Vol. I39, No. 1, FEBRUARY 1992 
master processor also executes the process which displays grey-level images of flows on a graphics monitor.

The speed-up of imaging frame rate when different numbers of slave processors are used is shown in Table 2 .

Table 2: Speed-up of imaging frame rate

\begin{tabular}{lll}
\hline No. of slave-processor & image frame/sec & speed-up \\
\hline 1 & 10.5 & 1.0 \\
2 & 20.3 & 1.93 \\
3 & 29.4 & 2.80 \\
\hline
\end{tabular}

The speed-up is almost linear. It can be predicted that, to achieve 50 frame/second imaging speed, at least 5 T800 slave processors should be used (if the graphics transputer is fast enough).

\section{Conclusions}

This paper has demonstrated the capability of a software tool facilitating algorithm development and sensor design for capacitive tomography systems.

The backprojection algorithm has been improved, producing images with better fidelity. The primary sensor has been optimised for a gas/oil two-component flow, minimising nonlinearity in capacitance measurements. A method for determining volume concentration using capacitance measurements has been proposed

Imaging speed can be significantly increased by using Inmos's new generation transputers, code-named $\mathrm{H} 1$. The H1 transputer retains compatibility with the $\mathrm{T} 800$ series, and is ten times faster in both processing and communication speeds. It can be anticipated that, using $\mathrm{H} 1$ transputers, a 100 frame-per-second imaging speed can be readily achieved. The software tool described in this paper will also benefit from the introduction of the $\mathrm{H} 1$ transputers, running ten times faster.

\section{Acknowledgment}

The authors acknowledge the Science and Engineering Research Council and the Department of Trade and Industry for supporting this work through a grant under the LINK scheme.

\section{References}

1 HUANG, S.M., PLASKOWSKI, A., XIE, C.G., and BECK, M.S Tomographic imaging of two-component flow using capacitance sensors', J. Phys. E, 1989, 22, (3), pp. 173-177

2 XIE, C.G., PLASKOWSKI, A., and BECK, M.S.: "8-electrode capacitance system for two-component flow identification. Part 1: Tomographic flow imaging; Part 2: Flow regime identification', IEE Proc. A, 1989, 136, (4), pp. 173-19

3 SALKELD, J.A.: 1991 PhD thesis, University of Manchester

4 THORN, R., HUANG, S.M., XIE, C.G., SALKELD, J.A., HUNT, A., and BECK, M.S.: 'Flow imaging for multi-component flow measurement', Flow Meas. Instrum., 1990, 1, (4), pp. 259-268

5 HUANG, S.M., XIE, C.G., THORN, R., SNOWDEN, D., and BECK, M.S.: 'Design of sensor electronics for electrical capacitance tomography', IEE Proc. G, 1992, 139, (1), pp. 83-88

6 SILVESTER, P.P., and FERRARI, R.L.: 'Finite element for electrical engineers' (New York: Cambridge University Press, 1990) 2nd Edition

7 HUGHES, T.J.R.: The finite element method - linear static and dynamic finite element analysis' (Prentice-Hall International, 1987)

8 'Transputer reference manual'. Inmos Ltd (New York: Prentice Hall, 1988)

9 'Transputer development system'. Inmos Ltd (New York: Prentice Hall, 1990) 2nd Edition

10 XIE, C.G., STOTT, A.L., PLASKOWSKI, A., and BECK, M.S 'Design of capacitance electrodes for concentration measurement of two-phase flow', Meas. Sci. Technol., 1990, 1, (1), pp. 65-78

11 KHAN, S., and ABDULLAH, F.: 'Computer-aided design of process tomography capacitance electrode system for flow imaging'. Proc. 5th Conf. Sensors and their applications, September 1991, Edinburgh, pp. 209-214

12 LOSHIN, D.: 'Making every bit count', Parallelogram, 1991, pp $12-14$

13 BOWLER, KC KENWAY, R.D. PAWLEY, G.S. ROWETH, D and WILSON, G.V.: 'An introduction to OCCAM 2 programming' (Studentlitteratur: Chartwell-Bratt, 1989) 2nd edition 